\title{
Chemical spots on the surface of the strongly magnetic Herbig Ae star HD 101412
}

\author{
S.P. Järvinen ${ }^{1, \star \star}$, S. Hubrig ${ }^{1}$, M. Schöller ${ }^{2}$, I. Ilyin ${ }^{1}$, T. A. Carroll ${ }^{1}$, and H. Korhonen ${ }^{3,4}$ \\ 1 Leibniz-Institut für Astrophysik Potsdam (AIP), An der Sternwarte 16, 14482 Potsdam, Germany \\ 2 European Southern Observatory, Karl-Schwarzschild-Str. 2, 85748 Garching, Germany \\ 3 Finnish Centre for Astronomy with ESO (FINCA), University of Turku, Väisäläntie 20, 21500 Piikkiö, Finland \\ 4 Dark Cosmology Centre, Niels Bohr Institute, Copenhagen University, Juliane Maries Vej 30, 2100, Copenhagen Ø, \\ Denmark
}

Received 2015, accepted 2016

Published online XXXX

\begin{abstract}
Key words stars: pre-main sequence — stars: individual (HD 101412) - stars: magnetic fields - stars: oscillations stars: variables: general

Due to the knowledge of the rotation period and the presence of a rather strong surface magnetic field, the sharp-lined young Herbig Ae star HD 101412 with a rotation period of $42 \mathrm{~d}$ has become one of the most well-studied targets among the Herbig Ae stars. High-resolution HARPS polarimetric spectra of HD 101412 were recently obtained on seven different epochs. Our study of the spectral variability over the part of the rotation cycle covered by HARPS observations reveals that the line profiles of the elements $\mathrm{Mg}, \mathrm{Si}, \mathrm{Ca}, \mathrm{Ti}, \mathrm{Cr}, \mathrm{Mn}, \mathrm{Fe}$, and $\mathrm{Sr}$ are clearly variable while He exhibits variability that is opposite to the behaviour of the other elements studied. Since classical Ap stars usually show a relationship between the magnetic field geometry and the distribution of element spots, we used in our magnetic field measurements different line samples belonging to the three elements with the most numerous spectral lines, Ti, Cr, and Fe. Over the time interval covered by the available spectra, the longitudinal magnetic field changes sign from negative to positive polarity. The distribution of field values obtained using $\mathrm{Ti}, \mathrm{Cr}$, and $\mathrm{Fe}$ lines is, however, completely different compared to the magnetic field values determined in previous low-resolution FORS 2 measurements, where hydrogen Balmer lines are the main contributors to the magnetic field measurements, indicating the presence of concentration of the studied iron-peak elements in the region of the magnetic equator. Further, we discuss the potential role of contamination by the surrounding warm circumstellar matter in the appearance of Zeeman features obtained using Ti lines.
\end{abstract}

Copyright line will be provided by the publisher

\section{Introduction}

Among the Herbig stars with detected magnetic fields, HD 101412 possesses the strongest magnetic field up to $3.5 \mathrm{kG}$ (Hubrig et al. 2010), followed by V380 Ori with a dipole field strength of about $2.1 \mathrm{kG}$ (Alecian et al. 2009). The presence of such a strong magnetic field on the surface of HD 101412 makes it a prime candidate for highresolution spectropolarimetric studies of the impact of the magnetic field on the physical processes occurring during stellar formation. Hubrig et al. (2009) tested a number of atmospheric models in the range: $T_{\text {eff }}=8000-11000 \mathrm{~K}$ and $\log g=4.0-4.05$ and obtained as a best fit $T_{\text {eff }}=10000 \mathrm{~K}$, $\log g=4.2-4.3$, and a $v \sin i$ value of about $5 \mathrm{~km} \mathrm{~s}^{-1}$. This result is in good agreement with the study of Guimarães et al. (2006), who published $T_{\text {eff }}=10000 \pm 1000 \mathrm{~K}, \log g=$ $4.1 \pm 0.4$, and $v \sin i=7 \pm 1 \mathrm{~km} \mathrm{~s}^{-1}$. A good fit was also obtained using the model $T_{\text {eff }}=9000 \mathrm{~K}$ and $\log g=4.0$, but

\footnotetext{
$\star$ Based on data obtained from the ESO Science Archive Facility under request MSCHOELLER 101895 (ESO programme Nos. 081.C-0410(A), 085.C-0137(A), and 187.D-0917(D)).

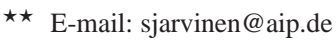

the synthetic spectrum for these parameters showed many narrow lines that did not appear in the observed UVES spectra (Hubrig et al. 2009). In their work, the authors mentioned that the spectrum of HD 101412 is heavily contaminated by weak circumstellar (CS) lines. The fundamental parameters, $T_{\text {eff }}=8300 \mathrm{~K}$ and $\log g=3.8$, and even lower projected rotation velocity, $v \sin i=3 \pm 2 \mathrm{~km} \mathrm{~s}^{-1}$, were determined by Cowley et al. (2010) using high-resolution HARPS spectra. According to their results, the photosphere is depleted in most refractory elements, while volatiles are normal or, in the case of nitrogen, overabundant with respect to the Sun. The authors suggested that the detected anomalous saturation of strong lines arises from heating of the upper atmospheric layers by infalling material from a disk. The overall abundance pattern may be related to those found for $\lambda$ Boo stars.

As of today, only about 20 late Herbig Be and Herbig Ae stars have been reported to possess large-scale organized magnetic fields (e.g. Hubrig et al. 2009, 2015, Alecian et al. 2013), using low- and/or high-resolution spectropolarimetric observations, and only for about half a dozen of this type of stars the detection of the magnetic field was 
achieved using both low- and high-resolution spectropolarimetric observations. Moreover, only for the two Herbig Ae stars HD 101412 and V380 Ori (Hubrig et al. 2011a; Alecian et al.2009), the magnetic field geometry has been constrained in previous studies.

Previously published mean longitudinal magnetic field measurements of the Herbig Ae star HD 101412 were based on low-resolution polarimetric spectra obtained with FORS 1/2 (FOcal Reducer low dispersion Spectrograph) mounted on the 8-m Antu telescope of the VLT (e.g. Wade et al. 2005, 2007, Hubrig et al. 2009, 2010, 2011a). In contrast to the results obtained by Wade et al. (2007) who measured a positive magnetic field of the order of $500 \mathrm{G}$ using hydrogen lines and simultaneously on the same spectra a negative magnetic field of the same order using metal lines, the studies of Hubrig et al. showed rather consistent results between the measurements obtained using the full spectrum including metal lines and those using hydrogen lines.

Combining photometric observations and measurements of the longitudinal magnetic field based on FORS 2 spectra obtained on 13 different epochs distributed over 62 days, Hubrig et al. (2011a) determined for HD 101412 a rotation period of $42.076 \mathrm{~d}$. The authors report that HD 101412 exhibits a single-wave variation in the longitudinal magnetic field during the stellar rotation cycle, which is usually considered as evidence for a dominant dipolar contribution to the magnetic field topology. Furthermore, high-resolution, high signal-to-noise UVES spectra and a few lower quality HARPS spectra revealed the presence of a few resolved magnetically split lines and a variation of the mean magnetic field modulus (Hubrig et al.2010). The Zeeman doublet Fe II at $\lambda 6149.258$ appeared resolved in the acquired spectra with the measured mean magnetic field modulus varying from 2.5 to $3.5 \mathrm{kG}$, while the mean quadratic magnetic field was found to vary in the range from 3.5 to $4.8 \mathrm{kG}$. The mean quadratic magnetic field is determined from the study of the second-order moments of the line profiles recorded in unpolarised light (that is, in the Stokes parameter I). Such an analysis is usually based on the consideration of samples of reasonably unblended lines in spectra (e.g. Mathys \& Hubrig 2006). Noteworthy, HD 101412 is presently the only Herbig Ae star for which the rotational Doppler effect was found to be small in comparison to the magnetic splitting of several spectral lines observed in unpolarised light.

High-resolution spectropolarimetric observations of HD 101412 on seven different epochs obtained using the HARPS (High Accuracy Radial velocity Planet Searcher) spectropolarimeter are publicly available in the archive (Prg. 187.D-0917) of the European Southern Observatory (ESO). In contrast to low-resolution spectropolarimetry, the high-resolution polarimetric spectra allow us to study in detail surface abundance inhomogeneities and, in particular, how different elements with different abundance distributions across the stellar surface sample the magnetic field. The same spectra were used by Järvinen et al. (2015),
Table 1 Logbook of the HARPS spectropolarimetric observations of the Herbig Ae star HD 101412.

\begin{tabular}{ccrc}
\hline \hline $\begin{array}{c}\text { HJD } \\
2450000+\end{array}$ & Phase $^{1}$ & SNR & $\begin{array}{c}\text { Exp. time } \\
{[\mathrm{s}]}\end{array}$ \\
\hline 6337.801 & 0.131 & 101 & $4 \times 700$ \\
6337.835 & 0.132 & 103 & $4 \times 700$ \\
6338.832 & 0.156 & 104 & $4 \times 700$ \\
6339.709 & 0.176 & 92 & $4 \times 600$ \\
6341.732 & 0.225 & 76 & $4 \times 600$ \\
6342.634 & 0.246 & 58 & $4 \times 600$ \\
6344.782 & 0.297 & 60 & $4 \times 600$ \\
\hline
\end{tabular}

1 The phases are based on the rotation period of $42.076 \mathrm{~d}$ determined by Hubrig et al. (2011a).

where magnetic field measurements using iron lines were presented along with the magnetic field measurements of two other sharp-lined Herbig Ae stars, HD 104237 and HD 190073. The emphasis in that study of HD 101412 was however put on the investigation of the impact of the circumstellar matter on the observed line profiles.

In Sect. 2, we report on the observations and data reduction, in Sect. 3 we present our search for spectral variability using lines belonging to different elements, and in Sect. 4 we describe the methods and results of our magnetic field measurements using samples of lines belonging to different elements. Finally, in Sect. 5 we discuss the significance of the obtained results for future directions in the studies of Herbig Ae stars.

\section{Observations and data reduction}

Seven spectropolarimetric observations were obtained with the HARPS polarimeter (HARPSpol; Snik et al. 2008) attached to ESO's $3.6 \mathrm{~m}$ telescope (La Silla, Chile) on the nights from 2013 February 14 to 21 . Among them, two individual observations were obtained during the first night on February 14. Although these two observations are obtained at approximately the same rotation phase, we consider them in our magnetic field study individually to learn about the limitations of our measurement methods. The seven HARPS archival spectra cover rotation phases from 0.131 to 0.297 . Each observation was split into four subexposures with an exposure time of 10-12 min, obtained with different orientations of the quarter-wave retarder plate relative to the beam splitter of the circular polarimeter. The achieved signal-to-noise ratio (SNR) in the final Stokes $I$ spectra, summed over the four subexposures, is rather low, accounting for a SNR between 58 and 104. In Table 1, the dates of observations are presented in the first column, followed by the rotation phase and the achieved signal-to-noise ratio. The exposure times are given in the last column. The spectra have a resolving power of about $R=115000$ and cover the spectral range $3780-6910 \AA$, with a small gap between 5259 and $5337 \AA$. The reduction and calibration of the archive spectra was performed using the HARPS data 
reduction software available at the ESO headquarter in Germany.

The normalization of the spectra to the continuum level consisted of several steps described in detail by Hubrig et al. (2013). The Stokes $I$ and $V$ parameters were derived following the ratio method described by Donati et al. (1997), and null polarization spectra were calculated by combining the subexposures in such a way that the intrinsic source polarization cancels out, yielding a diagnostic null $N$ spectrum. These steps ensure that no spurious signals are present in the obtained data (e.g. Ilyin 2012).

\section{Spectral variability}

Not much is known about the presence of chemical spots on the surface of magnetic Herbig Ae stars, although they are usually considered as progenitors of classical magnetic Ap stars, which generally display a surface inhomogeneous distribution of different elements. In Ap stars with largescale organized, predominantly dipolar magnetic fields, the non-uniformly distributed chemical elements show a certain symmetry with respect to the magnetic field configuration. As an example, rare earth element spots are frequently identified close to magnetic poles, while iron peak element spots tend to concentrate in the vicinity of the magnetic equator (e.g. Elkin et al.2010). The previous studies of HD 101412 by Hubrig et al. (2010, 2011a) using a sample of iron lines revealed line variations of equivalent widths, radial velocities, line widths, line asymmetries, and mean magnetic field modulus. Given the rather low SNR of the HARPS spectra, to study the spectral variability, we employed the Least-Squares Deconvolution (LSD) technique allowing us to achieve a much higher SNR in the LSD spectra. The details of this technique can be found in the work of Donati et al. (1997).

The absence of $\delta$ Scuti-like pulsations was already discussed in the previous work of Järvinen et al. (2015) where the authors verified that no changes in the line profile shape or radial velocity shifts are present in the obtained spectra on a short-time scale. This step was of importance since pulsations are known to have an impact on the analysis of the presence of a magnetic field and its strength (e.g. Schnerr et al. 2006, Hubrig et al.2011b).

In the previous studies by Hubrig et al. (2011a, 2012), the available spectra had an imperfect phase coverage over the rotation cycle, especially in the phase range between 0.2 and 0.5 (see Fig. 5 in Hubrig et al. 2011a). The polarimetric HARPS spectra allow us now to partly close this gap covering the phases from 0.131 to 0.297 . For the study of the variability of the line profiles belonging to different elements over different rotation phases, we tried to choose only lines with a low magnetic sensitivity, i.e. lines with low Landé factors. The reason for this is that the magnetic field in HD 101412 is rather strong, and magnetic intensification, i.e. differential broadening of spectral lines having different magnetic sensitivities is expected (e.g. Hubrig et al. 1999).

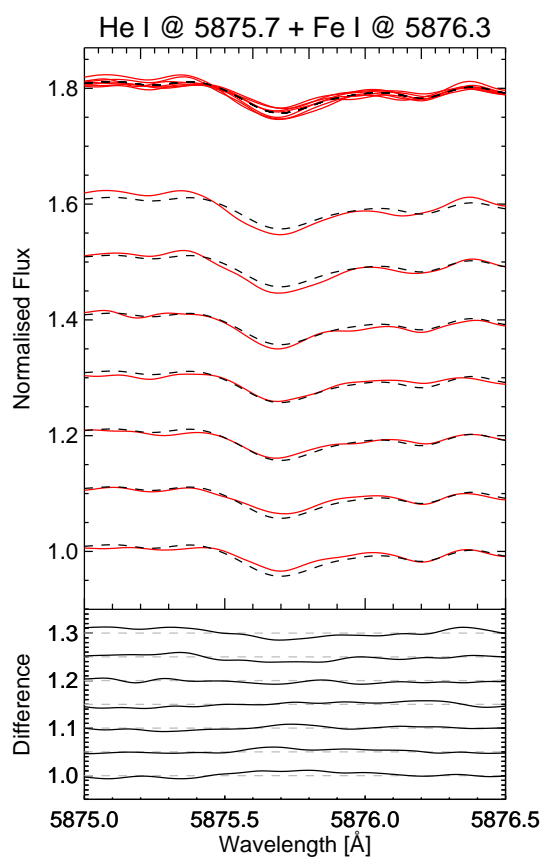

Fig. 2 Comparison of the Stokes I profiles for the He I $\lambda 5875.7$ line recorded with HARPS on seven different epochs. As in Fig. 1, the black dashed lines present the average profiles. The overplotted profiles are shown on the top, whereas the lower panel displays the differences between the Stokes $I$ profiles obtained for the individual exposures and the average Stokes $I$ profile.

In Fig. 1, for different elements, we present a comparison of Stokes $I$ profiles for spectral lines with low Landé factors obtained on seven different epochs. The studied spectral lines are Ca I $\left(\lambda 4226.7 \AA, g_{\text {eff }}=1.000\right)$, Tі II $(\lambda 4417.7 \AA$, $\left.g_{\text {eff }}=0.795\right)$, Cr II $\left(\lambda 4634.1 \AA, g_{\text {eff }}=0.508\right), \operatorname{MnI}(\lambda$ $\left.4034.5 \AA, g_{\text {eff }}=0.827\right), F_{\text {I }}\left(\lambda 4071.7 \AA, g_{\text {eff }}=0.679\right)$, and Fe II $\left(\lambda 4508.3 \AA, g_{\text {eff }}=0.500\right)$. Due to the presence of a rather strong noise in the HARPS spectra, before plotting the line profiles, we have slightly smoothed them using a Gaussian filter with a width of $0.01 \AA$. A clear decrease of line intensities is detected in all studied profiles in the time interval between the first and the last observing night. The variability displayed by the iron lines shows a trend similar to the iron line variability already discussed in the previous studies by Hubrig et al. (2010, 2011a). Since we also detect variations of the lines belonging to the elements $\mathrm{Ca}$, Ti, $\mathrm{Cr}$, and $\mathrm{Mn}$ as a function of the rotation phase, we conclude that all these elements are inhomogeneously distributed on the surface of HD 101412. The previously reported variability of a few other elements, such as $\mathrm{He}, \mathrm{Si}, \mathrm{Mg}$, and $\mathrm{Sr}$, by Hubrig et al. (2011a, 2012) is also detected in our HARPS spectra. In Figs. 2 and 3, we present the behaviour of the profiles of $\mathrm{He}$ I $\lambda$ 5876, the oxygen quintet $\mathrm{O}$ I at $\lambda 6157, \mathrm{Si}$ II $\lambda$ 6371.4, the $\mathrm{Mg}$ II doublet at $\lambda$ 4481, and $\mathrm{Sr}$ II at $\lambda$ 4215.5. For these elements, due to the sparseness of the available lines in the spectrum of HD 101412, it was not possible to 


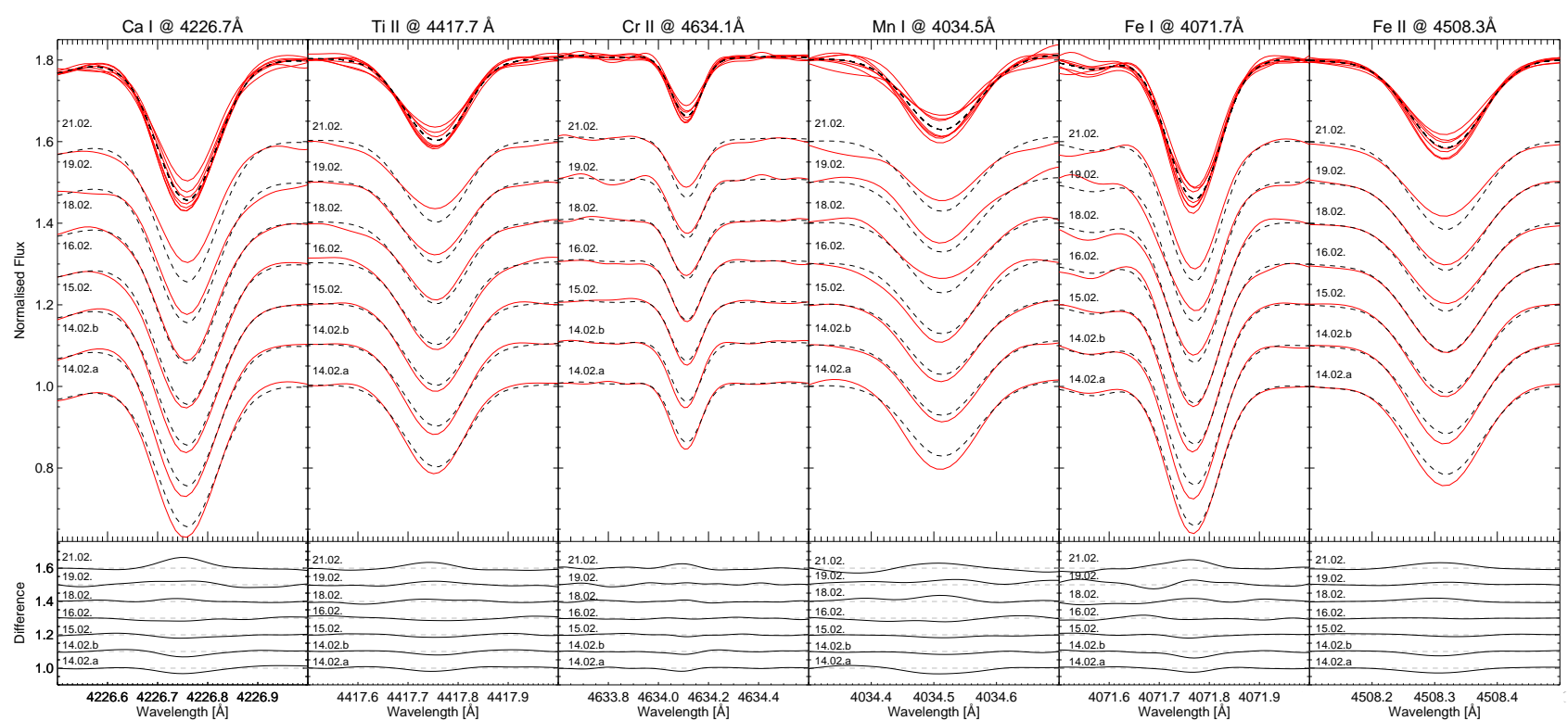

Fig. 1 Comparison of Stokes I profiles for spectral lines belonging to the elements $\mathrm{Ca}, \mathrm{Ti}, \mathrm{Cr}, \mathrm{Mn}$, and $\mathrm{Fe}$ recorded with HARPS on seven different epochs. The black dashed lines present the average profiles. The overplotted profiles are shown on the top, whereas the lower panels display the differences between the Stokes I profiles obtained for the individual exposures and the average Stokes $I$ profile.

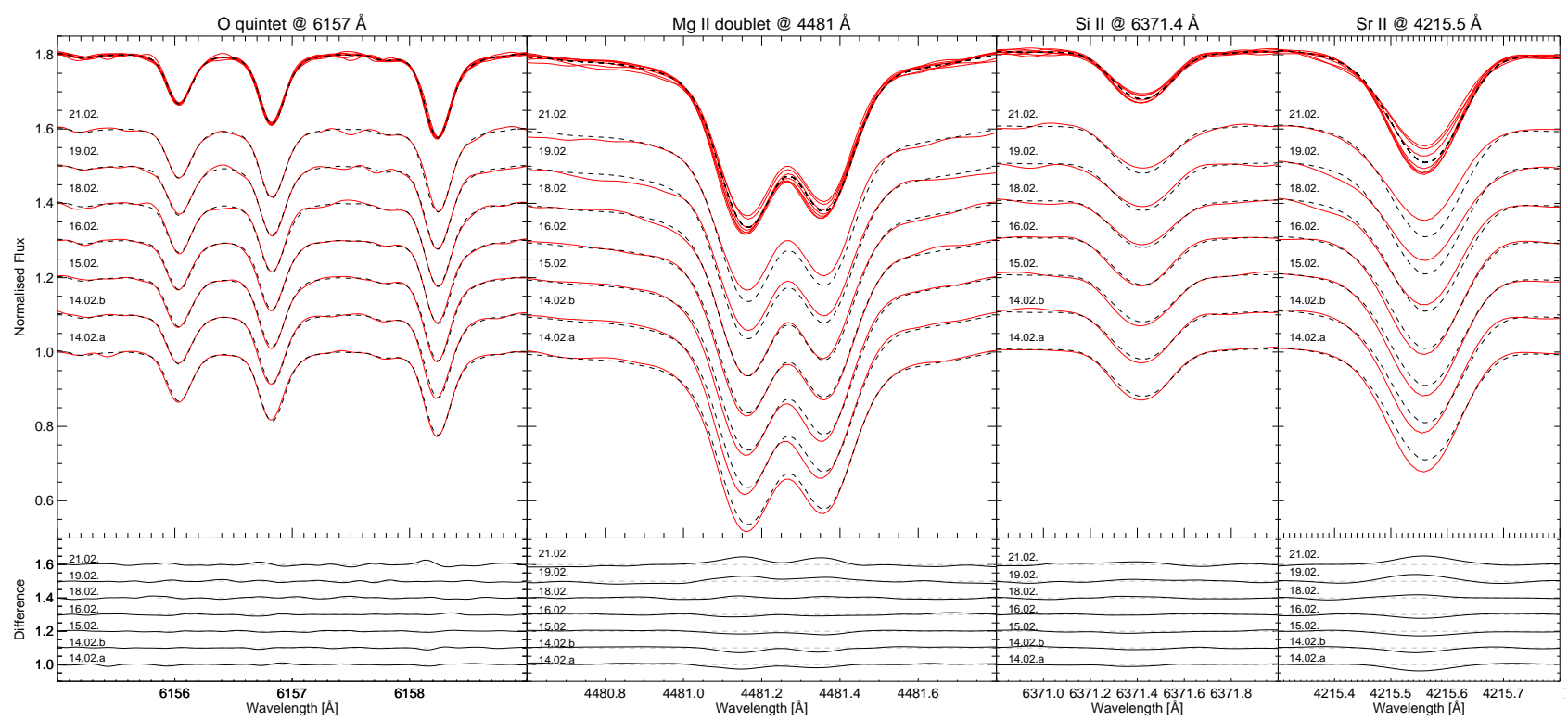

Fig. 3 Comparison of Stokes I profiles for spectral lines belonging to the elements $\mathrm{O}, \mathrm{Mg}$, $\mathrm{Si}$, and $\mathrm{Sr}$ recorded with HARPS on seven different epochs. The black dashed lines present the average profiles. As in Fig. 1, the overplotted profiles are shown on the top, whereas the lower panels display the differences between the Stokes I profiles obtained for the individual exposures and the average Stokes $I$ profile.

choose lines with low Landé factors. Among them, we detect that He exhibits variability that is opposite to the behaviour of the other studied elements: the line intensity is increasing between the first and the last observing nights. Also oxygen behaves differently, showing no changes of the line profiles over the same time interval.

\section{Magnetic field measurements}

Generally, in classical magnetic Ap stars with inhomogeneous surface element distribution, the actual measured magnetic field strength is dependent on the surface distribution of the elements from whose lines the measurements are made (e.g. Elkin et al.2010). Since the lines of different elements with different abundance distributions across the 
stellar surface sample the magnetic field in a different manner, we selected for our measurements four individual line masks containing lines belonging to the elements with the most rich spectra in HD 101412. In our analysis, we have used the LSD method to boost up the signal in our HARPS spectra. This technique combines line profiles (using the assumption that line formation is similar in all lines) centred at the position of the individual lines given in the line mask and scaled according to the line strength and sensitivity to a magnetic field (i.e. to a Landé factor). The resulting average profiles (Stokes $I$, Stokes $V$, and $N$ ) obtained by combining several lines, yield an increase in signal-to-noise ratio and therefore in sensitivity to polarization signatures. Only lines stronger than $10 \%$ of the continuum considering only natural broadening have been selected. Following the study based on Fe I lines presented by Järvinen et al. (2015) the line masks for the LSD code were created for atmospheric parameters $T_{\text {eff }}=8300 \mathrm{~K}$ as well as $T_{\text {eff }}=10000 \mathrm{~K}$ and $\log g=3.8$ using the VALD database (e.g. Kupka et al. 2000). Since line blends affect the individual lines, i.e. occurring at different positions within the line profile and having different strength, only unblended lines were chosen. The first pair of masks was consisting of 86 and $50 \mathrm{Ti}$ II lines for $T_{\text {eff }}=8300 \mathrm{~K}$ and $T_{\text {eff }}=10000 \mathrm{~K}$, respectively, and the second pair of 31 and $21 \mathrm{Cr}$ II lines, again for the two temperatures. The results based on $52 \mathrm{Fe}$ II lines forming at $T_{\text {eff }}=8300 \mathrm{~K}$ were already published in Järvinen et al. (2015), and therefore only Fe II lines (47 in all) forming at $T_{\text {eff }}=10000 \mathrm{~K}$ are considered here. The forth line mask included the lines of all these elements together forming at $T_{\text {eff }}=10000 \mathrm{~K}$. No line mask for $\mathrm{He}, \mathrm{Ca}, \mathrm{Mn}$, or any rare earth element was created because of the low number of lines suitable for magnetic field measurements. Due to the especially low SNR at the blue and red ends of the HARPS spectra, the spectral lines used for the computation of the LSD profiles have been selected in the wavelength region from 4000 to $6500 \AA$. The mean longitudinal magnetic field is evaluated by computing the first-order moment of the Stokes $V$ profile according to Mathys (1989).

The resulting mean LSD Stokes $I$, Stokes $V$, and diagnostic $N$ profiles obtained for different line samples and different effective temperatures are presented in Figs. 40 Distinct Zeeman features are detected at all epochs in all LSD Stokes $V$ spectra. Only for the sample of $\mathrm{Cr}$ II lines on the first three epochs do the Stokes $V$ spectra not show any conspicuous feature, due to the fact that the noise at these epochs has a stronger impact on the measurements caused by a rather low number of lines in the line mask.

For all observations, the null spectra appear flat, indicating the absence of spurious polarization. Using the false alarm probability (FAP; Donati et al. 1992) in the region corresponding to the whole Stokes $I$ line profile (velocity range $\pm 15 \mathrm{~km} \mathrm{~s}^{-1}$, we obtain definite magnetic field detections with FAP $<10^{-8}$ at all epochs for the samples of Ti II, Fe II, and the sample consisting of all lines together. For the sample of $\mathrm{Cr}$ II lines, definite detections are achieved only for the four last epochs. According to Donati et al. (1992), an FAP smaller than $10^{-5}$ can be considered as a definite detection, while $10^{-5}<F A P<10^{-3}$ is considered as a marginal detection. The measurement results are summarized in Table 2 .

The inspection of the LSD profiles calculated for each sample presented in Figs. 44 9 reveals that the amplitude of the Zeeman features increases towards the latest epochs and that the majority of these features have a shape typical to that observed in classical magnetic stars during crossover, i.e. in the phase interval when the magnetic field changes its polarity (e.g. Hubrig et al. 2014).

Järvinen et al. (2015) already mentioned the fact that rather large differences in the shape of Zeeman features are observed between the Fe I and $\mathrm{Fe}$ II samples on the first four epochs. In the LSD Stokes $V$ profile obtained for $\mathrm{Fe}$ I lines, they observed a distinct feature appearing as a second maximum in the red wing of the Zeeman profile. Furthermore, Stokes $V$ profiles for neutral iron were slightly shifted to the blue by about $4 \mathrm{~km} \mathrm{~s}^{-1}$. A similar behaviour of the LSD Stokes $V$ profile was also observed for the sample containing the lines of different elements together and explained by the high proportion of neutral iron lines in this sample. As is shown in Figs. 4 and 5, also the LSD Stokes $V$ profiles obtained for the Ti II lines share the same behaviour. Nothing can be concluded about the behaviour of the LSD Stokes $V$ profiles for $\mathrm{Cr}$ II at the first four epochs due to the rather low SNR.

From our experience with the work on magnetic fields in classical Ap stars exhibiting chemical spots, we know that usually neutral and ionized $\mathrm{Fe}$ behave very similar, i.e. the magnetic field measurements for them are almost identical, indicating that these stars do not show temperature spots. Admittedly, the issue whether Herbig Ae stars exhibit temperature spots typical to those observed in T Tauri stars is currently unexplored. On the other hand, the velocity information, i.e. the presence of small shifts in the LSD Stokes $V$ profiles obtained for the neutral $\mathrm{Fe}$ and $\mathrm{Ti}$ II lists indicates that we probably observe a contamination by the surrounding warm circumstellar (CS) matter in the form of a wind. Such a scenario was already discussed by Järvinen et al. (2015) and would be in agreement with the previous finding of Hubrig et al. (2009) who reported a strong contamination of the UVES spectra of this star by weak lines of neutral and ionized iron (see their Fig. 6), where the lines of neutral iron are by far more numerous than those of ionized iron.

To decrease the impact of the contamination by the CS contribution, Järvinen et al. (2015) studied the behaviour of the LSD Stokes $V$ profiles calculated for a sample of neutral iron lines forming at a significantly higher effective temperature, i.e. assuming $T_{\text {eff }}=10000 \mathrm{~K}$, and could show that at a significantly higher $T_{\text {eff }}$ distinct features in the red wings of the Zeeman profiles and velocity shifts completely disappear. However, the LSD Stokes $V$ profiles calculated for the first epochs for the sample of Ti II lines formed at different $T_{\text {eff }}$ still resemble each other rather well, i.e. the profiles 


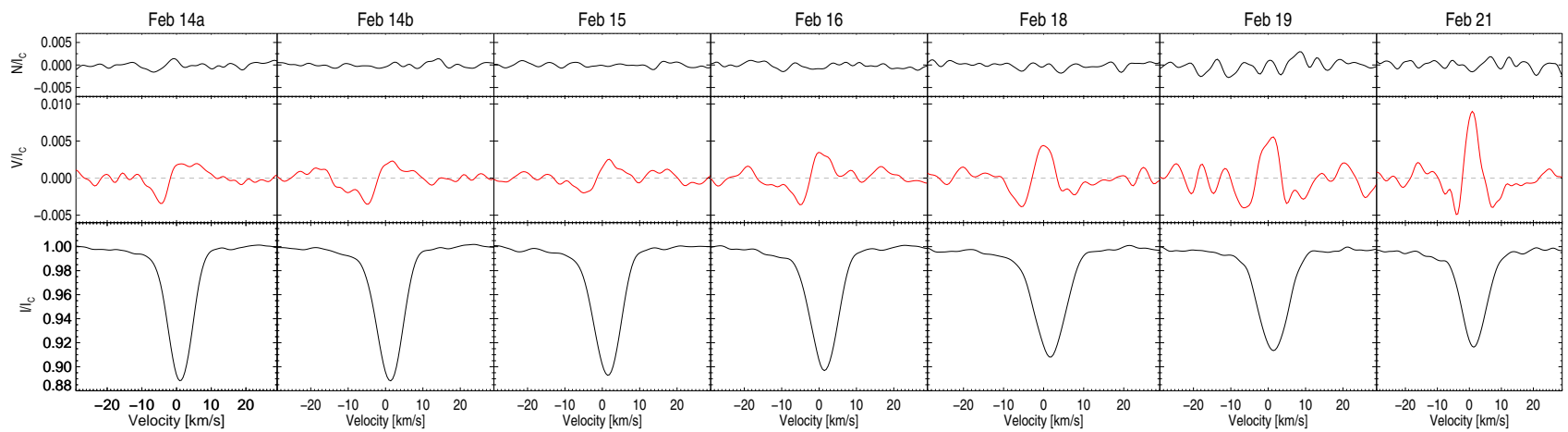

Fig. 4 The LSD $I, V$, and $N$ spectra obtained for HD 101412 on seven different epochs using a sample of 86 Ti II lines forming at $T_{\text {eff }}=8300 \mathrm{~K}$.

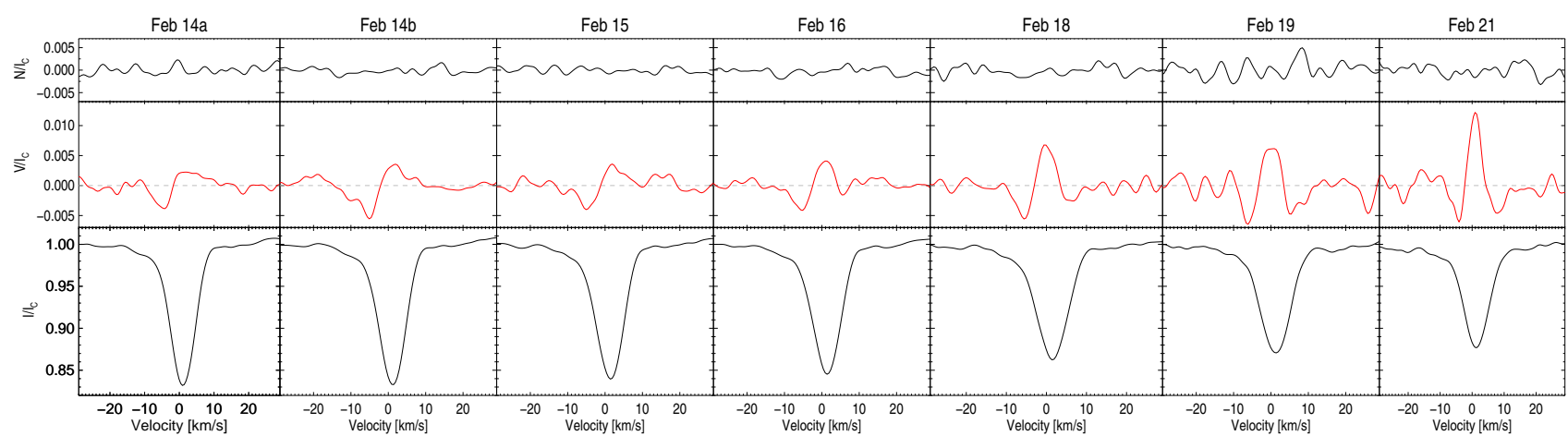

Fig.5 As Fig. 团but using a sample of $50 \mathrm{Ti}$ II lines forming at $T_{\text {eff }}=10000 \mathrm{~K}$.

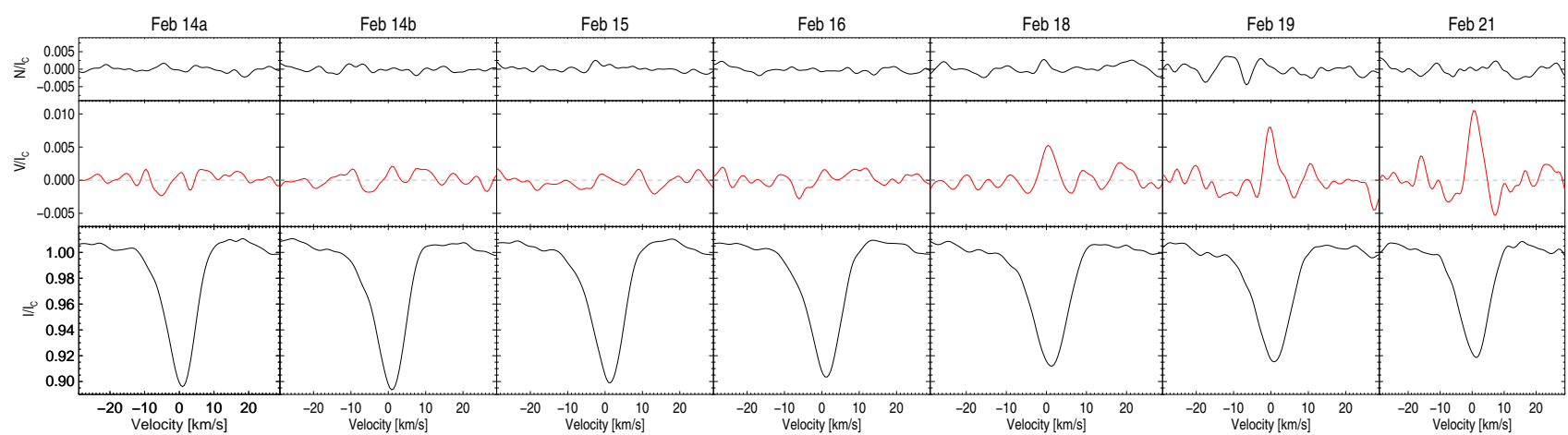

Fig. 6 As Fig. 4, but using a sample of $31 \mathrm{Cr}$ II lines forming at $T_{\text {eff }}=8300 \mathrm{~K}$.

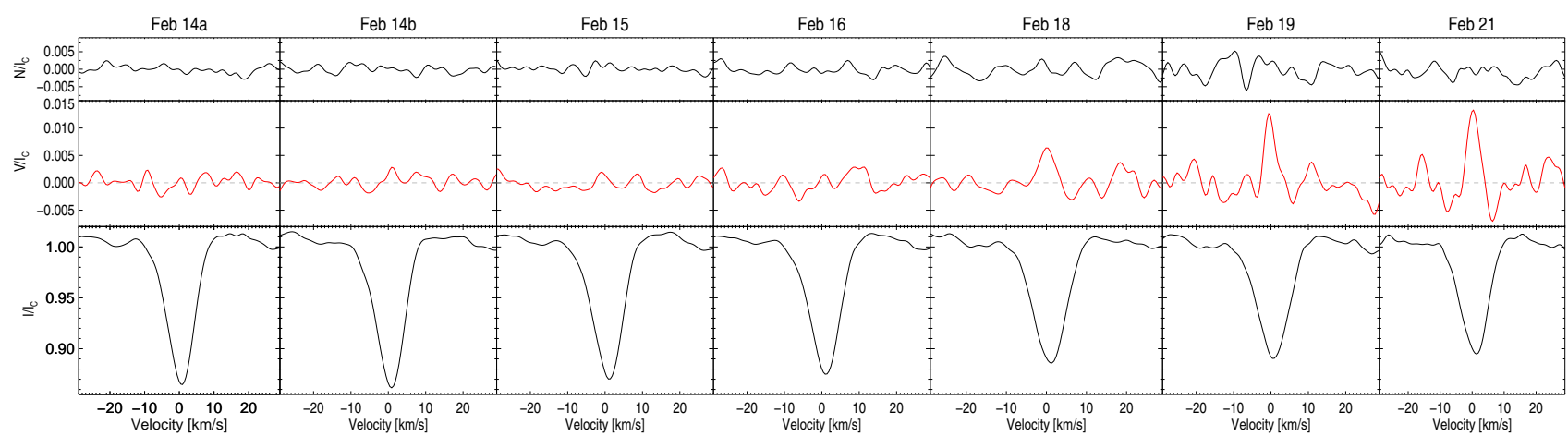

Fig. 7 As Fig. 4, but using a sample of $21 \mathrm{Cr}$ II lines forming at $T_{\text {eff }}=8300 \mathrm{~K}$. 


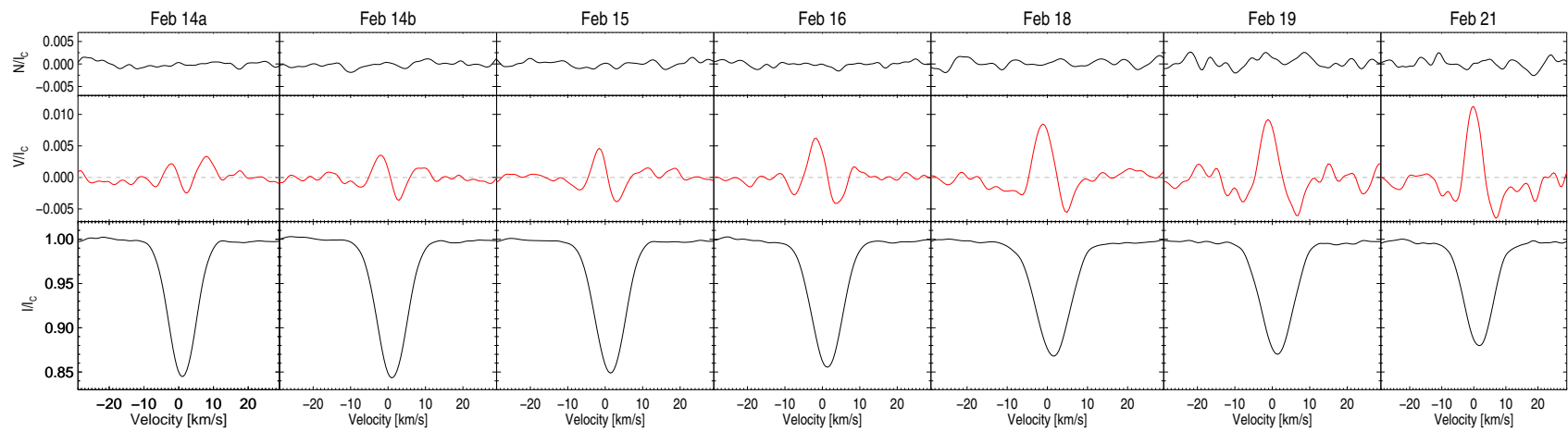

Fig. 8 As Fig. 4 but using a sample of $47 \mathrm{Fe}$ II lines forming at $T_{\text {eff }}=10000 \mathrm{~K}$.

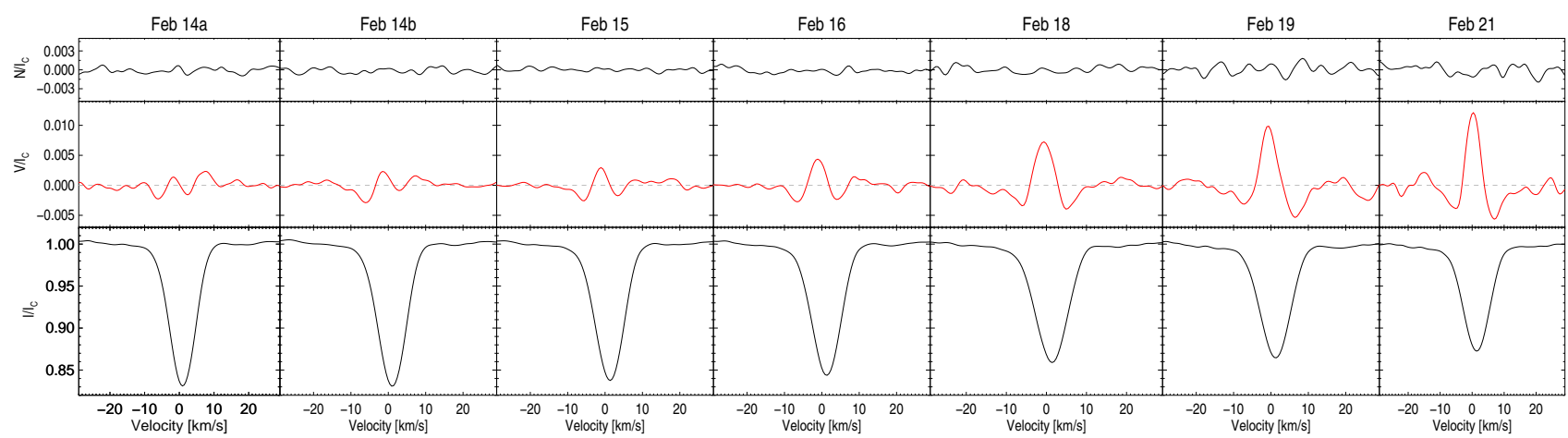

Fig. 9 As Fig. 4, but using all lines forming at $T_{\text {eff }}=10000 \mathrm{~K}$.

Table 2 For each sample created for $T_{\text {eff }}=10000 \mathrm{~K}$, the measured longitudinal magnetic field and the signal-to-noise value achieved in the LSD spectra are presented. The average Landé factor used in the LSD measurements is also indicated. The FAP is always less than $10^{-8}$.

\begin{tabular}{|c|c|c|c|c|c|c|c|c|c|}
\hline $\begin{array}{c}\text { HJD } \\
2450000+\end{array}$ & Phase & SNR & $\begin{array}{c}\left\langle B_{\mathrm{z}}\right\rangle_{\mathrm{Ti}} \text { II } \\
{[\mathrm{G}]}\end{array}$ & SNR & $\begin{array}{c}\left\langle B_{\mathrm{z}}\right\rangle_{\mathrm{Cr} \text { II }} \\
{[\mathrm{G}]}\end{array}$ & SNR & $\begin{array}{c}\left\langle B_{\mathrm{z}}\right\rangle_{\mathrm{Fe} \mathrm{II}} \\
{[\mathrm{G}]}\end{array}$ & SNR & $\begin{array}{c}\left\langle B_{\mathrm{z}}\right\rangle_{\mathrm{All}} \\
{[\mathrm{G}]}\end{array}$ \\
\hline & & \multicolumn{2}{|c|}{$\bar{g}_{\mathrm{eff}}=1.04$} & \multicolumn{2}{|c|}{$\bar{g}_{\mathrm{eff}}=1.15$} & \multicolumn{2}{|c|}{$\bar{g}_{\mathrm{eff}}=1.09$} & \multicolumn{2}{|c|}{$\bar{g}_{\mathrm{eff}}=1.13$} \\
\hline 6337.801 & 0.131 & 670 & $-99 \pm 18$ & 302 & & 859 & $-8 \pm 11$ & 1215 & $-69 \pm 8$ \\
\hline 6337.835 & 0.132 & 749 & $-122 \pm 18$ & 350 & & 1203 & $5 \pm 14$ & 1547 & $-82 \pm 9$ \\
\hline 6338.832 & 0.156 & 866 & $-156 \pm 24$ & 375 & & 1333 & $58 \pm 16$ & 1835 & $-72 \pm 12$ \\
\hline 6339.709 & 0.176 & 458 & $-115 \pm 20$ & 295 & $-87 \pm 35$ & 725 & $62 \pm 18$ & 1053 & $-49 \pm 10$ \\
\hline 6341.732 & 0.225 & 377 & $-18 \pm 29$ & 280 & $29 \pm 54$ & 701 & $81 \pm 22$ & 773 & $-14 \pm 17$ \\
\hline 6342.634 & 0.246 & 287 & $48 \pm 41$ & 198 & $-53 \pm 91$ & 470 & $82 \pm 20$ & 632 & $41 \pm 29$ \\
\hline 6344.782 & 0.297 & 355 & $51 \pm 45$ & 218 & $-20 \pm 83$ & 688 & $70 \pm 19$ & 639 & $85 \pm 24$ \\
\hline
\end{tabular}

remain almost unchanged. Such a behaviour found for Ti II lines can possibly be explained by the fact that the sample contains a large number of $\mathrm{Ti}$ II lines with a low excitation potential and thus the impact of the CS contamination still persists.

To obtain additional characteristics of the magnetic field in HD 101412, we used the moment technique developed by Mathys (e.g. 1991). Importantly, this technique allows us not only the estimation of the mean longitudinal magnetic field, but also to prove the presence of crossover effect and of the quadratic magnetic field. It was already shown in the past that depending on the magnetic field geometry, even stars with rather weak longitudinal magnetic fields can exhibit strong crossover effects and kG quadratic fields (see e.g. Mathys 1995; Landstreet \& Mathys 2000; Mathys \&
Hubrig 1995, 2006). The moment technique was applied to the same sample of unblended $45 \mathrm{Fe}$ II lines as selected for the LSD analysis. These measurements are presented in Table 3 For each line in this sample, the measured shifts between the line profiles in the left- and right-hand circularly polarized HARPS spectra are used in a linear regression analysis in the $\Delta \lambda$ versus $\lambda^{2} g_{\text {eff }}$ diagram, following the formalism discussed by Mathys (1991, 1994). Similar to the LSD results, the longitudinal magnetic field shows negative polarity during the first epochs and positive polarity during the last epochs. The values for the field strength are in good agreement with those obtained using the LSD method within the measurement uncertainties. No significant magnetic fields could be determined from the null spectra. The measured quadratic magnetic field varies from about $3 \mathrm{kG}$ to 
Table 3 Magnetic field measurements of the Herbig Ae star HD 101412 using the moment technique. All quoted errors are $1 \sigma$ uncertainties.

\begin{tabular}{crrrc}
\hline \hline $\begin{array}{c}\text { HJD } \\
2450000+\end{array}$ & SNR & \multicolumn{1}{c}{$\begin{array}{c}\left\langle B_{\mathrm{z}}\right\rangle_{\mathrm{Fe}_{\text {II }}} \\
{[\mathrm{G}]}\end{array}$} & $\begin{array}{c}\left\langle B_{\text {xover }}\right\rangle \\
{[\mathrm{G}]}\end{array}$ & $\begin{array}{c}\left\langle B_{\mathrm{q}}\right\rangle \\
{[\mathrm{G}]}\end{array}$ \\
\hline 6337.801 & 101 & $-62 \pm 28$ & $33 \pm 69$ & $3087 \pm 204$ \\
6337.835 & 103 & $-37 \pm 27$ & $4 \pm 73$ & $2999 \pm 198$ \\
6338.832 & 104 & $-7 \pm 27$ & $70 \pm 64$ & $3153 \pm 205$ \\
6339.709 & 92 & $36 \pm 29$ & $115 \pm 83$ & $3103 \pm 257$ \\
6341.732 & 76 & $70 \pm 35$ & $350 \pm 93$ & $3232 \pm 287$ \\
6342.634 & 58 & $160 \pm 56$ & $425 \pm 131$ & $3381 \pm 403$ \\
6344.782 & 60 & $141 \pm 54$ & $511 \pm 130$ & $3579 \pm 430$ \\
\hline
\end{tabular}

$3.6 \mathrm{kG}$ over the rotation phase interval from 0.131 to 0.297 . The crossover effect at a significance level larger than $3 \sigma$ is detected at the last three epochs in the rotation phase interval from 0.225 to 0.297 .

\section{Discussion}

In Fig. 10, we present the measurement results obtained for different line masks using the LSD method and the results obtained using the moment technique overplotted with the previous FORS 2 measurements. In the upper panel we present the measurements obtained by Hubrig et al. (2011a) based on FORS 2 observations together with our new measurements using Fe II lines formed at $T_{\text {eff }}=10000 \mathrm{~K}$ and previous measurements using Fe $\mathrm{I}$ forming at the same temperature, already presented in the work of Järvinen et al. 2015). The distribution of the field values obtained using HARPSpol spectra is completely different compared to the magnetic field values determined in previous low-resolution FORS 2 spectra, where hydrogen Balmer lines are the main contributors to the magnetic field measurements. Similar differences between the distribution of field values obtained using HARPSpol and FORS 2 are also detected in the measurements using samples of $\mathrm{Ti}$ II and $\mathrm{Cr}$ II lines presented in the middle panel. Also the measurements using the moment technique presented in the bottom panel deviate significantly from the FORS 2 measurements. For all considered samples, the amplitude of the magnetic field strength is significantly lower than that observed in FORS 2 spectra.

In contrast to low-resolution FORS 2 measurements, the HARPS measurements are carried out on metal lines, which in classical magnetic Ap stars usually exhibit surface chemical spots. Our variability study of lines belonging to the elements $\mathrm{Mg}, \mathrm{Si}, \mathrm{Ca}, \mathrm{Ti}, \mathrm{Cr}, \mathrm{Mn}, \mathrm{Fe}$, and $\mathrm{Sr}$ indicates the presence of changes in the line profiles over the part of the rotation cycle covered by HARPS observations, hinting at the presence of chemical spots on the stellar surface. Furthermore, He seems to exhibit variability that is opposite to the behaviour of the other studied elements. The shape of the LSD Stokes $V$ profiles displaying crossover effect indicates that we observe a change from negative to positive polarity, i.e. both negative and positive magnetic poles are

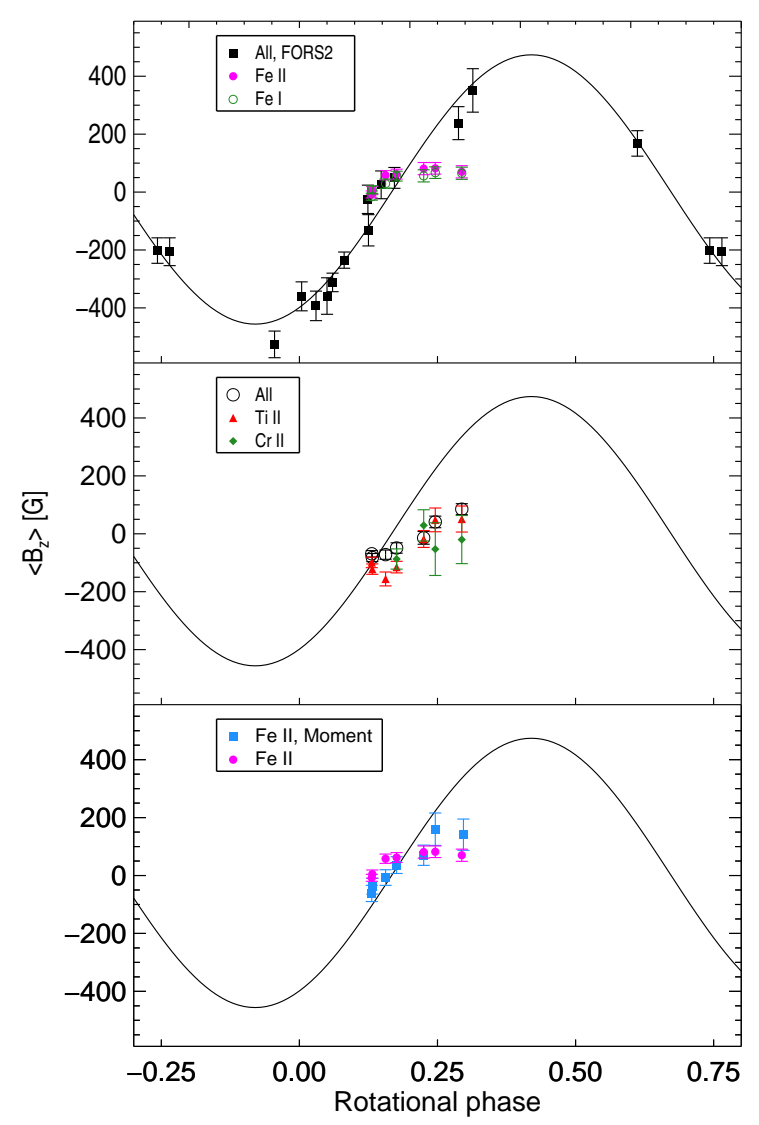

Fig. 10 Phase diagram for the longitudinal magnetic field measurements carried out using low-resolution FORS 2 spectropolarimetric observations and high-resolution HARPS spectropolarimetric observations. The fit on all three panels is from Hubrig et al. (2011a). Top panel: Black squares are from Hubrig et al. (2011a) based on FORS 2 observations. Pink filled circles represent LSD measurements from Fe II lines that form at $T_{\text {eff }}=10000 \mathrm{~K}$, whereas green open circles are LSD measurements from Fe I forming also at the same temperature (values are presented in Järvinen et al. 2015). Middle panel: Open black circles show the LSD measurements obtained using all lines that form at $T_{\text {eff }}=10000 \mathrm{~K}$. Red filled triangles show Ti II line LSD measurements, and Cr II line LSD measurements are indicated with dark green filled diamonds. Bottom panel: Blue squares show measurements from Fe II lines using the moment technique and pink filled circles are, as in the top panel, Fe II LSD measurements.

visible. The obtained result that the amplitude of the magnetic field strength measured on metal lines is significantly lower than that observed in FORS 2 spectra is a well-known phenomenon frequently observed in Ap stars if the magnetic field measurements are carried out using line samples belonging to elements concentrated far from magnetic poles. For instance, in a number of studies of such stars, iron peak elements appear enhanced along the magnetic equator (e.g. Nesvacil et al. 2012). We note that the assumption of the 
presence of concentration of these elements in the region of the magnetic equator, similar to the iron peak element distribution appearing in Ap stars, would explain the amplitude and the shape of the observed Zeeman features displaying crossover effect. The results of our measurements of the mean longitudinal magnetic field based on different samples are comparable within the measurement accuracies using the LSD method.

Järvinen et al. (2015) revealed significant differences between the appearance of Zeeman features using the sample of Fe II lines and Fe I lines formed at $T_{\text {eff }}=8300 \mathrm{~K}$. This difference disappears if the LSD profiles are calculated for the sample of Fe I lines at $T_{\text {eff }}=10000 \mathrm{~K}$. Järvinen et al. discuss that the differences between the measurements using iron line samples can be caused by temperature inhomogeneities or by a contamination by the surrounding warm CS matter. If temperature spots are indeed present on the surface of Herbig Ae stars, then it could be suggested that $\mathrm{Fe}_{\mathrm{I}}$ lines are formed in somewhat cooler spots. On the other hand, the presence of small shifts of Stokes $V$ profiles to shorter wavelengths by about $4 \mathrm{~km} \mathrm{~s}^{-1}$ obtained for neutral Fe indicates that we probably observe a contamination by the surrounding warm CS matter in the form of a wind. The observed similarity between the shapes of Zeeman features in the Stokes $V$ spectra of Fe I at $T_{\text {eff }}=10000 \mathrm{~K}$ and Fe II support the suggested scenario. In this work we show for the first time that similar distinct features and shifts appear also in Zeeman profiles calculated for the sample of Ti II lines.

Magnetic fields in a few Herbig Ae stars were already discovered a decade ago (e.g. Hubrig et al. 2004, 2006 , 2015, Wade et al.2005), but our understanding of the interaction between the central stars, their magnetic fields, and their protoplanetary discs is still very limited. It is not clear yet whether the majority of Herbig Ae stars do possess weak magnetic fields, which are not detected due to the rather low accuracy of the magnetic field measurements in previous studies. To gain better knowledge of typical magnetic field strengths in Herbig Ae/Be stars, Hubrig et al. (2015) recently compiled all magnetic field measurements reported in previous spectropolarimetric studies. The obtained density distribution of the rms longitudinal magnetic field values reveals that only very few stars have fields stronger than $200 \mathrm{G}$, and half of the sample possesses magnetic fields of about $100 \mathrm{G}$ and less. Noteworthy, in the currently largest high-resolution spectropolarimetric survey of the magnetic field in these stars by Alecian et al. (2013) with 132 measurements for 70 Herbig stars, the measurement accuracy is worse than $200 \mathrm{G}$ for $35 \%$ of the measurements, and for $32 \%$ of the measurements it is between 100 and $200 \mathrm{G}$, i.e. only $33 \%$ of the measurements showed a measurement accuracy of better than $100 \mathrm{G}$.

We note also that without acquiring high-quality spectropolarimetric material, it will not be possible to decide whether there is a fundamental difference between magnetic and non-magnetic Herbig stars. Since the number of Herbig stars with a firmly detected magnetic field is currently small, fundamental questions related to the accretion geometry remain unanswered. The detection of very weak longitudinal magnetic fields in these stars put into question our current understanding of the magnetospheric accretion process in intermediate-mass pre-main sequence stars, as they indicate that their magnetic fields are by far weaker than those measured in their lower mass counterparts, the classical T Tauri stars, usually possessing $\mathrm{kG}$ magnetic fields. In this respect, the presence of a strong magnetic field in HD 101412 and its slow rotation make this object a most favourable candidate for studies of the role of the magnetic field in the star formation process.

Acknowledgements. We would like to thank G. Lo Curto for his support with the reduction of the HARPS polarimetric spectra. SPJ acknowledges the support by the Deutsche Forschungsgemeinschaft, grant JA 2499/1 -1.

\section{References}

Alecian, E., Wade, G.A., Catala, C., et al. 2009, MNRAS, 400, 354 Alecian, E., Wade, G.A., Catala, C., et al. 2013, MNRAS, 429, 1001

Carroll, T.A., Strassmeier, K.G., Rice, J.B., \& Künstler, A. 2012, A\&A, 548, A95

Cowley, C.R., Hubrig, S., González, J.F., \& Savanov, I. 2010, A\&A, 523, A65

Donati, J.-F., Semel, M., \& Rees, D.E. 1992, A\&A, 265, 669

Donati, J.-F., Semel, M., Carter, B.D., et al. 1997, MNRAS, 291, 658

Elkin, V.G., Mathys, G., Kurtz, D.W., et al. 2010, MNRAS, 402, 1883

Guimarães, M.M., Alencar, S.H.P., Corradi, W.J.B., \& Vieira, S.L.A. 2006, A\&A, 457, 581

Hubrig, S., Castelli, F., \& Wahlgren, G.M. 1999, A\&A, 346, 139

Hubrig, S., Schöller, M., \& Yudin, R.V. 2004, A\&A, 428, L1

Hubrig, S., Yudin, R.V., Schöller, M., \& Pogodin, M.A. 2006, A\&A, 446, 1089

Hubrig, S., Stelzer, B., Schöller, M., et al. 2009, A\&A, 502, 283

Hubrig, S., Schöller, M., Savanov, I., et al. 2010, Astr. Nachr., 331, 361

Hubrig, S., Mikulášek, Z., González, J.F., et al. 2011a, A\&A, 525, L4

Hubrig, S., Ilyin, I., Briquet, M., et al. 2011b, A\&A, 531, L20

Hubrig, S., Castelli, F., González, J.F., et al. 2012, A\&A 542, A31

Hubrig, S., Schöller, M., Ilyin, I., \& Lo Curto, G. 2013, Astr. Nachr., 334, 1093

Hubrig, S., Carroll, T.A., González, J.F., et al. 2014, MNRAS 440, L6

Hubrig, S., Carroll, T.A., Schöller, M., \& Ilyin, I. 2015, MNRAS, 449, L118

Ilyin I. 2012, Astr. Nachr., 333, 213

Järvinen, S.P., Carroll, T.A., Hubrig, S., et al. 2015, A\&A, 584, A15

Kallinger, T., Zwintz, K., \& Weiss, W. 2008, A\&A, 488, 279

Kupka, F.G., Ryabchikova, T.A., Piskunov, N.E., et al. 2000, Baltic Astr., 9, 590

Landstreet, J. \& Mathys, G. 2000, A\&A, 359, 213

Mathys, G. 1989, Fundamentals of Cosmic Physics, 13, 143

Mathys, G. 1991, A\&AS, 89, 121

Mathys, G. 1994, A\&AS, 108, 547

Mathys, G. 1995, A\&A, 293, 746 
Mathys, G.\& Hubrig, S. 1997, A\&AS, 124, 475

Mathys, G. \& Hubrig, S. 2006, A\&A, 453, 699

Nesvacil, N., Lüftinger, T., Shulyak, D., et al. 2012, A\&A, 537, A151

Schnerr, R.S., Verdugo, E., Henrichs, H.F., \& Neiner, C. 2006, A\&A, 452, 969

Snik, F., Jeffers, S., Keller, C., et al. 2008, SPIE, 7014, 0 O

Wade, G.A., Drouin, D., Bagnulo, S., et al. 2005, A\&A, 442, L31

Wade, G.A., Bagnulo, S., Drouin, D., et al. 2007, MNRAS, 376, 1145 